\title{
Homogeneous magnetic fields in fully anisotropic string cosmological backgrounds
}

\author{
Massimo Giovannini \\ Institute for Theoretical Physics, University of Lausanne, BSP-Dorigny, CH-1015 Switzerland
}

\begin{abstract}
We present new solutions of the string cosmological effective action in the presence of a homogeneous Maxwell field with pure magnetic component. Exact solutions are derived in the case of spaceindependent dilaton and vanishing torsion background. In our examples the four dimensional metric is either of Bianchi-type III and $\mathrm{VI}_{-1}$ or Kantowski-Sachs.
\end{abstract}

The solutions of the low-energy string cosmological effective action are, by their nature anisotropic [1]. In this context, it is important to analyze the role of the different possible sources of anisotropy in the low energy string effective action. An interesting and motivated source of anisotropy is represented by pure magnetic fields [2]. Exact string cosmological solutions (containing a magnetic field) were recently found for Bianchi-type I background geometries in the case of a vanishing torsion background and for a space-independent dilaton field [2]. It was shown that while the anisotropy can increase for some time, the string tension corrections (combined with the post big-bang evolution) are likely to force the anisotropy to decay.

The purpose of our paper is very simple: we want to generalize the Bianchi-type I solutions of the string cosmological effective action to the case where the homogeneous magnetic field is contained in more general homogeneous (but still anisotropic) metrics. In the context of the low-energy string effective action, the analysis of Bianchi-type classes has been performed (in the absence of any electromagnetic background) by Gasperini and Ricci [3].

In the Bianchi-type I case the homogeneous magnetic (or electric) background is not constrained by the group theoretical properties of the (Abelian) isometry group. In spite of this observation the solutions of the string cosmological effective action are not trivial in the sense that they cannot be found in the usual general relativity literature. The reason is that the dilaton field $\phi$ couples directly to the kinetic term of the Abelian gauge field. In four space-time dimensions such a coupling can be expressed as $e^{-\phi} F_{\mu \nu} F^{\mu \nu}$ where $\mu, \nu=0, \ldots 3$ and $F_{\mu \nu}$ is the Abelian gauge field strength. Bianchi models (different from Bianchi-type I) are characterized by a non-Abelian isometry group: the Killing vectors (leaving invariant the three-dimensional spatial submanifold) do not commute. Therefore, we face the problem of accommodating an Abelian gauge field in a geometry whose algebraic structure is non-Abelian. The consequence of this remark is that not all non-Abelian isometry groups will be able to support a homogeneous electromagnetic field. More precisely, only Bianchi-type I, II, III, $\mathrm{VI}_{-1}$ and $\mathrm{VII}_{0}$ can accommodate a homogeneous magnetic field polarized along a fixed direction [何. This observation holds in the case of general relativity (where the coupling of the metric to the Maxwell fields is dictated by the equivalence principle) and also in our case provided the dilaton field is space-independent and provided we deal with pure magnetic field (i.e. the homogeneous components of the electric fields are all vanishing).

In order to show this point in detail let us look at the relevant Maxwell's equations. Then we will specify the background geometry which is uniquely characterized by the group theoretical properties of the algebra of the Killing vectors. Finally, we will be able to show which Bianchi-type metrics can (or cannot) be compatible with the presence of a homogeneous (Abelian) magnetic field. Let us start from the Maxwell's equations and from the related Bianchi identities

$$
\partial_{\mu}\left(e^{-\phi} \sqrt{-g} F^{\mu \nu}\right)=0, \quad \partial_{\mu}\left(\sqrt{-g} \tilde{F}^{\mu \nu}\right)=0,
$$

where $\tilde{F}^{\mu \nu}$ is the dual field strength..

In the absence of electric fields and for the case of space-independent dilaton background the gauge field strengths can be written, in the language of differential forms, as

$$
F=\frac{1}{2} B_{i} \epsilon_{i j k} \sigma^{j} \wedge \sigma^{k},{ }^{*} F=-B_{i} \sigma^{i} \wedge \sigma^{0},
$$

where $\sigma^{0}=d t$ and $\sigma^{i}=R_{j}^{i} \omega^{j}$ form the Cartan basis of one-form; the two-form $F$ represents the electromagnetic field and ${ }^{*} F$ its dual. The matrices $R_{i}^{j}$ depend only on time. If we take, for instance Cartesian coordinates as local basis the relation between the exterior derivative of the invariant basis is given by :

$$
d \omega^{i}=\frac{1}{2} C_{j k}{ }^{i} \omega^{j} \wedge \omega^{k}
$$

where $C_{j k}{ }^{i}$ are the structure constants of the three dimesional isometry group which appear in the commutation relations of the Killing vectors. For the case of Bianchitype I we have that $C_{j k}{ }^{i}$ are identically zero (i.e. the isometry group is Abelian). For the other Bianchi-type geometries the structure constants are not all vanishing (i.e. the isometry group is non-Abelian).

By now expressing Eqs. (11) in the invariant basis of the $\omega^{i}$ and by recalling that the dilaton field is only time dependent we obtain a system of three linear equations, namely 


$$
\begin{aligned}
& \dot{Z}_{a}=0, \quad M_{j} C_{a b}{ }^{j} \epsilon_{c a b}=0, \\
& Z_{j} C_{a j}{ }^{j}=0,
\end{aligned}
$$

where the over-dot denotes differentiation with respect to $t$ and where

$$
\begin{aligned}
Z_{a} & =\frac{1}{2} B_{i} \epsilon_{i j k} \epsilon_{a b c} R_{b}^{j} R_{c}^{k}, \\
M_{j} & =-\frac{1}{2} R_{j}^{i} B_{i} .
\end{aligned}
$$

The solution of the first of Eqs. (何) gives $Z_{a}=q_{a}$ where $q_{a}$ are constant vectors. Therefore Eqs. (4)-(5) can be written as

$$
Z_{a}=q_{a}, q_{a} C_{j a}^{j}=0, \quad M_{j} C_{a b}^{j}=0 .
$$

It is clear from these expressions that in the case where all the $C_{j k}{ }^{i}=0$ the magnetic field components are not constrained since all the equations (7) are identically statisfied for any vector $B_{i}$ and $q_{a}$. In the Bianchi-type II case we have that the only non-vanishing structure constant is $C_{23}{ }^{1}=1$. In this case the first two equations of (7) are always satisfied, whereas the third of Eqs. (7) constrains the magnetic field to vanish in one specific direction (i.e. the magnetic field can only have two independent components). Similarly, in the Bianchi-type III case the only non-vansihing structure constant is $C_{23}^{2}=1$ and therefore both, the second and the third equation of (7) will be non trivially satisfied. Thus in Bianchi-type III models the magnetic field will have only one independent component: the other two will have to vanish for the compatibility with the non-Abelian structure of the isometry group. In the Bianchi-type IV and V cases the structure constants are, respectively

$$
\begin{aligned}
& C_{13}{ }^{1}=C_{23}{ }^{1}=C_{23}{ }^{2}=1, \quad \mathrm{IV}, \\
& C_{13}{ }^{1}=C_{23}{ }^{2}=1, \quad \mathrm{~V} .
\end{aligned}
$$

From the second of Eq. (7) we see that, in the Bianchitype IV and V, $q_{3}=0$. Moreover form the third of Eq. (7) we see that $B_{k}=0$. Therefore, in the Bianchi-type IV and V the isometry group is only compatible with a vanishing (pure) magnetic field.

In the Bianchi-type VI and VII the structure constants are, respectively,

$$
\begin{aligned}
& C_{13}{ }^{1}=1, \quad C_{23}^{2}=h, \quad \mathrm{VI}, \\
& C_{32}{ }^{1}=C_{13}{ }^{2}=1, \quad C_{23}{ }^{2}=h, \text { VII. }
\end{aligned}
$$

In the case of Bianchi-type VI a magnetic field can be only present (though with only one independent component) if $h=-1$. Only in this case one can consistently satisfy the second of Eqs. (7). In the Bianchi-type VII geometry, for the same reason, a magnetic field (with one independent component) can be present only if $h=0$. Finally in the Bianchi-type VIII and IX metrics the structure constants are, respectively

$$
\begin{aligned}
& C_{23}{ }^{1}=C_{12}{ }^{3}=C_{13}{ }^{2}=1, \quad \text { VIII, } \\
& C_{i j}{ }^{k}=\epsilon_{i j k}, \quad \text { IX. }
\end{aligned}
$$

In these two last cases we can see that the third equation of (7) implies that $B_{i} R^{i} j=0$ which is identically satisfied only in the absence of any magnetic background.

In conclusion we showed that if the dilaton field only depends upon time and if the electric field is absent only the Bianchi-type I, II, III, $\mathrm{VI}_{-1}, \mathrm{VII}_{0}$ are compatible with the presence of a magnetic background. In the Bianchitype I case no constraint on the components of the magnetic field is present. In the other Bianchi-type models the magnetic field is always constrained. In the Bianchitype IV, V, $\mathrm{VI}_{h \neq 1}, \mathrm{VII}_{h \neq 0}$, VIII, IX cases we cannot accommodate a pure (Abelian) magnetic field.

What we discussed, up to now is only a sufficient condition in order to accommodate a magnetic background with time dependent dilaton in a Bianchi-type geometry. Our analysis does not guarantee the existence of explicit (exact) solutions.

In the following, as an example, we want to present few exact solutions containing both a magnetic field, a time-dependent dilaton and a background geometry with non-Abelian isometry group. The action 7 we ought to study is

$$
S=-\int d^{4} x \sqrt{-g} e^{-\phi}\left[R+g^{\alpha \beta} \partial_{\alpha} \phi \partial_{\beta} \phi+\frac{1}{4} F_{\mu \nu} F^{\mu \nu}\right]
$$

whose associated equations of motion can be written as

$$
\begin{aligned}
& R-g^{\alpha \beta} \partial_{\alpha} \phi \partial_{\beta} \phi+2 g^{\alpha \beta} \nabla_{\alpha} \nabla_{\beta} \phi=-\frac{1}{4} F_{\alpha \beta} F^{\alpha \beta}, \\
& R_{\mu}^{\nu}+\nabla_{\mu} \nabla^{\nu} \phi+\frac{1}{2} F_{\mu \alpha} F^{\nu \alpha}=0 \\
& \nabla_{\mu}\left[e^{-\phi} F^{\mu \nu}\right]=0 .
\end{aligned}
$$

where $\nabla_{\mu}$ denotes covariant differentiation with respect to the background metric. Notice that in writing the previous action we assumed zero central charge deficit. This means that the six internal dimensions are assumed to be compactified with constant radius. For sake of simplicity we choose to parametrize our line element as

$$
d s^{2}=d t^{2}-a^{2}(t) d x^{2}-b^{2}(t) e^{2 \lambda x} d y^{2}-c^{2}(t) d z^{2} .
$$

Notice that for $\lambda=-1$ we have the Bianchi III line element, whereas the case $\lambda=-2$ we get the Bianchi $\mathrm{VI}_{-1}$ case. In the metric of Eq. (15) the components of Eqs. (12)-(14) can be found by direct projection on

\footnotetext{
${ }^{*}$ We work in string units and we set the string tension $\alpha^{\prime}$ equal to one
} 
the spatial vielbein. Care must be taken, however, in solving the generalized Maxwell equation (14). Eq. (14) together with the Bianchi identities imply, in the absence of sources, that a pure magnetic field can be accommodated along the $z$ axis so that $F_{\alpha \beta} F^{\alpha \beta}=2 B^{2} / a^{2} b^{2}(B$ is a constant). With these specifications and by defining the shifted time 周 derivative of the dilaton $\dot{\bar{\phi}}=\dot{\phi}-(H+F+G)$ together with the expansion rates along the three different spatial directions (i.e. $H=\dot{a} / a, F=\dot{b} / b$ and $G=\dot{c} / c)$ we get to

$$
\begin{aligned}
& 2 \ddot{\bar{\phi}}-\dot{\bar{\phi}}^{2}-\left(H^{2}+F^{2}+G^{2}\right)+2 \frac{\lambda^{2}}{a^{2}}+\frac{B^{2}}{2 a^{2} b^{2}}=0, \\
& \ddot{\bar{\phi}}-\left(H^{2}+F^{2}+G^{2}\right)=0, \\
& H \dot{\bar{\phi}}-\dot{H}+\frac{\lambda^{2}}{a^{2}}+\frac{B^{2}}{2 a^{2} b^{2}}=0, \\
& F \dot{\bar{\phi}}-\dot{F}+\frac{\lambda^{2}}{a^{2}}+\frac{B^{2}}{2 a^{2} b^{2}}=0, \\
& -\frac{\lambda}{a^{2}}(H-F)=0,
\end{aligned}
$$

In order to solve exactly the previous system we can define a new time coordinate, namely, $d t=e^{-\bar{\phi}} d \eta$. By denoting with a prime the derivatives with respect to $\eta$ we can re-write the system of equations (16)-(20) as

$$
\begin{aligned}
& \mathcal{G}^{\prime}=0, \quad \mathcal{H}=\mathcal{F}, \\
& \mathcal{H}^{\prime}=\lambda^{2} a^{2} c^{2} e^{-2 \phi}+\frac{B^{2}}{2} c^{2} e^{-2 \phi}, \\
& \phi^{\prime \prime}+\phi^{\prime 2}-2 Q \phi^{\prime}-Q^{\prime}+P=0, \\
& Q(\eta)=\frac{d \ln \sqrt{-g}}{d \eta}, \quad P=2(2 \mathcal{H} \mathcal{F}+\mathcal{F} \mathcal{G})
\end{aligned}
$$

where the prime denotes now the derivative with respect to $\eta$ and where $\mathcal{H}=a^{\prime} / a, \mathcal{F}=b^{\prime} / b, \mathcal{G}=c^{\prime} / c$. By linearly combining the previous equations and by defining $\Phi=\phi-\ln c$ the equation for $\Phi$

$$
\Phi^{\prime \prime}=\frac{B^{2}}{2} e^{-2 \Phi},
$$

can be integrated directly twice with the result that

$$
\Phi=\ln \left[\frac{\delta}{\gamma} \cosh \gamma\left(\eta-\eta_{0}\right)\right]
$$

where $\delta=B / \sqrt{2} ; \gamma$ and $\eta_{0}$ are integration constants. By now going to Eq. (22) and by making the ansatz $a(\eta)=e^{\Phi} f(\eta)$ we get an equation for $f(\eta)$

$$
\left(\frac{f^{\prime}}{f}\right)^{\prime}=\lambda^{2} f^{2}
$$

\footnotetext{
$\dagger$ The over-dot denotes derivation with respect to the cosmic time coordinate $t$.
}

which, again, can be directly integrated with the result that

$$
f(\eta)=-\frac{\beta}{\lambda\left|\sinh \beta\left(\eta-\eta_{0}\right)\right|}
$$

Putting all our results together we obtain that

$$
\begin{aligned}
& \phi(\eta)=\ln c_{0}+\mathcal{G}_{0} \eta+\ln \left[\frac{\delta}{\gamma} \cosh \gamma\left(\eta-\eta_{0}\right)\right], \\
& a(\eta)=-\frac{\beta \delta}{\lambda \gamma} \frac{\cosh \gamma\left(\eta-\eta_{0}\right)}{\left|\sinh \beta\left(\eta-\eta_{0}\right)\right|}, \\
& c(\eta)=c_{0} e^{\mathcal{G}_{0} \eta} .
\end{aligned}
$$

By requiring the consistency of this solution with the constraint equation we find that $2 \beta^{2}=\gamma^{2}+\mathcal{G}_{0}^{2}$.

It is interesting to notice that the solution we just obtained can be related to the solution of the same set of Eqs. (12) $-(14)$ in a Kantowski-Sachs [5] metric with a magnetic field oriented along the radial coordinate. The Kantowski-Sachs line element can be written as

$$
d s^{2}=d t^{2}-m^{2}(t) d r^{2}-n^{2}(t)\left[d \theta^{2}+A^{2}(\theta) d \chi^{2}\right] .
$$

where $A(\theta)=\sin \theta, \theta, \sinh \theta$. If a magnetic field directed along the radial direction is present, then, the equations of motion (13)-(14) will become

$$
\begin{aligned}
& \mathcal{M}^{\prime}=0, \\
& \mathcal{N}^{\prime}=-k n^{2} m^{2} e^{-2 \phi}+\frac{B^{2}}{2} m^{2} e^{-2 \phi}, \\
& \phi^{\prime \prime}+\phi^{\prime 2}-2 Q \phi^{\prime}-Q^{\prime}+P=0, \\
& Q(\eta)=(\mathcal{M}+2 \mathcal{N}), \quad P=2\left(\mathcal{M N}+2 \mathcal{N}^{2}\right),
\end{aligned}
$$

where $k=+1,0, \quad-1$ if, respectively, $A(\theta)=$ $\sin \theta, \theta, \sinh \theta$. This system can be integrated with the same techniques we discussed in the Bianchi case. Notice, moreover, that in the case $k=-1$ the analogy with the solutions (29)-31) is complete.

We want now to discuss the cosmic-time evolution of our solutions. Let us focus, for instance, on the Bianchitype II case $(\lambda=-1)$. On the basis of our results we can consistently choose $\beta=\gamma=1$. The consistency relation $2 \beta^{2}=\gamma^{2}+\mathcal{G}_{0}^{2}$ implies that $\mathcal{G}_{0}=1$ in string units. The relation between $\eta$ and the cosmic time coordinate can be obtained by direct integration of $a^{2} c e^{-\phi} d \eta=d t$. The result is

$$
\eta(t)=\ln \left[-\frac{\delta}{t}+\sqrt{1+\frac{\delta^{2}}{t^{2}}}\right] .
$$

We want, in particular, to analyze our solutions for $t<$ 0 . The solutions given in Eqs. (29)-(31) can then be expressed as

$$
a(t)=\frac{\sqrt{t^{2}+\delta^{2}}}{\delta},
$$




$$
\begin{aligned}
& c(t)=\left(\frac{\delta}{t}+\sqrt{\frac{\delta^{2}}{t^{2}}+1}\right)^{-1}, \\
& \phi(t)=\ln \left[\frac{\sqrt{1+\frac{\delta^{2}}{t^{2}}}}{\frac{\delta}{t}+\sqrt{1+\frac{\delta^{2}}{t^{2}}}}\right] .
\end{aligned}
$$

In order to write the solutions in this form we also have chosen $c_{0}=1$ and $\eta_{0}=0$. The choice $\eta_{0}=0$ simply translates in the origin the curvature singularity which appears clearly in $F=\dot{c} / c$. For $t<0$ the dilaton coupling grows. The scale factor $a(t)$ contracts to a minimal value determined by $\delta$, i.e. by the value of the magnetic field in string units. The scale factor $c(t)$ expands towards a singularity at $t=0$.

It is finally interesting to compute the shear parameter in the case we just described. The shear parameter measures the degree of isotropization of a given anisotropic solution [6]. We define the shear parameter, in our case , as

$$
r(t)=\frac{H-G}{H+2 G} .
$$

Using eqs. (38)-(39) we find that

$$
r(t)=\frac{\sqrt{t^{6}+\delta^{2} t^{4}}-\delta^{3}-\delta t^{2}}{2 \sqrt{t^{6}+\delta^{2} t^{4}}+\delta^{3}+\delta t^{2}} .
$$

By expanding the above expression in Taylor series around $t=0_{-}$we have that

$$
r(t)=-1+\frac{\delta+2}{\delta^{2}} t^{2}+\mathcal{O}\left(t^{3}\right)
$$

namely we see that the share parameter grows, a behavior similar to the one discussed in the Bianchi-type I case [2]. This behavior is then not surprising and it is due to the fact that, in our discussion, we did not take into account the string tension corrections to the low-energy action. If string tension corrections are (naively) included the shear parameter is forced to decrease.

In conclusion we discussed the possible inclusion of a pure (Abelian) magnetic field in the low-energy string effective action. We found that, in the absence of any torsion background, a magnetic field and a time-dependent dilaton field can be simultaneously accommodated in Bianchi-type I, II, III, VI -1, VII $_{0}$ backgrounds. As an example of our considerations we found new exact solutions of the low-energy string effective action in the Bianchitype III, $V I_{-1}$ and Kantowski-Sachs cases. We also discussed the physical properties of the obtained solutions and we argued that they are not qualitatively different from the ones deduced in the Bianchi-type I background [2].
[1] G. Veneziano, Phys. Lett. B 265, 287 (1991); M. Gasperini and G. Veneziano, Astropart. Phys. 1, 317 (1993).

[2] M. Giovannini, Phys. Rev. D 59, 123518 (1999); Phys. Rev. D 59, 063503 (1999).

[3] M. Gasperini and R. Ricci, Class.Quant.Grav.12, 677 (1995); M. Gasperini, R. Ricci and G. Veneziano, Phys. Lett. B 319, 438 (1993).

[4] L. P. Hugston and K. Jacobs, Astrophys. J. 160, 147 (1970).

[5] R. Kantowski and P. K. Sachs. J. Math. Phys. 7, 443 (1966).

[6] Ya. B. Zeldovich and I. D. Novikov, The Structure and evolution of the Universe (Chicago University Press, Chicago, 1971), Vol2. 